\title{
Gutenberg Richter and Characteristic Earthquake behavior in Simple Mean-Field Models of Heterogeneous Faults
}

\author{
Karin Dahmen and Deniz Ertaş̧ \\ Lyman Laboratory of Physics, Harvard University, Cambridge, Massachusetts, 02138 \\ Yehuda Ben-Zion \\ Department of Earth Sciences, Univ. of Southern CA, Los Angeles, CA, 90089-0740
}

(September 11, 2018)

\begin{abstract}
The statistics of earthquakes in a heterogeneous fault zone is studied analytically and numerically in a mean field version of a model for a segmented fault system in a three-dimensional elastic solid [1,2]. The studies focus on the interplay between the roles of disorder, dynamical effects, and driving mechanisms. A two-parameter phase diagram is found, spanned by the amplitude of dynamical weakening (or "overshoot") effects $\epsilon$ and the normal distance $L$ of the driving forces from the fault. In general, small $\epsilon$ and small $L$ are found to produce Gutenberg-Richter type power law statistics with an exponential cutoff, while large $\epsilon$ and large $L$ lead to a distribution of small events combined with characteristic system-size events. In a certain parameter regime the behavior is bistable, with transitions back and forth from one phase to the other on time scales determined by the fault size and other model parameters. The implications for realistic earthquake statistics are discussed.
\end{abstract}

PACS numbers: 91.30.Px, 05.40.+j, 62.20.Mk, 68.35.Rh 


\section{INTRODUCTION}

The statistics of earthquakes has been a subject of research for a long time. One spectacular feature is the wide range of observed earthquake sizes, spanning over ten decades in earthquake moment magnitude (which is defined to scale as the logarithm of the integral of slip along the fault during the earthquake [3]). Gutenberg and Richter [3] found in the 50's that the size distribution of regional earthquakes follows a power law over the entire range of observed events. The exponent $b$ of the power law distribution appears to be universal, i.e. it is approximately the same (within statistical errors and possible secondary dependency on the tectonic domain) for all studied regions. This type of power law distribution is called the "Gutenberg Richter" distribution. Recently, enough data has been collected to extract statistics on individual systems of earthquake faults, or more precisely on systems of narrow fault zones. Interestingly, it was found that the distribution of earthquake magnitudes may vary substantially from one fault system to another. In particular, Wesnousky and coworkers [4] found that fault systems with highly irregular geometry, such as the San Jacinto fault zone in California, which have many offsets and branches, display "power law" statistics over the whole range of observed magnitudes. Not all fault systems, however, display a power law distribution on all scales up to the largest earthquakes. The available data [4] indicate that fault systems with more regular geometry (presumably generated progressively with increasing cumulative slip) such as the San Andreas fault in California display power law distributions only for small events, which occur in the time intervals between roughly quasi-periodic earthquakes of a much larger "characteristic" size which rupture the entire fault. There are practically no observed earthquakes of intermediate magnitudes on such geometrically regular fault systems. Distributions of this type are called the "characteristic earthquake" distribution.

In previous work [1,2] it was demonstrated that a class of simple models of ruptures along a heterogeneous fault zone displays both types of behavior. The universal power law scaling behavior of earthquake statistics was seen to be due to an underlying critical point, 
which becomes mean-field-like for fault geometries with more than two spatial dimensions. In the limit of weak dynamical effects, the mean-field approximation to the 2 dimensional fault provides a more appropriate approximation than, for example, traditionally studied one dimensional approximations to the models. In fact, exact results for the scaling exponents (up to logarithmic corrections) could be obtained from mean-field theory. The reason is that the elastic stresses along the fault are effectively long range (decaying like the inverse cube of the distance), such that in two and higher dimensions the fluctuations due to interaction with other points on the fault decrease as the fault size is increased - on long length scales the behavior becomes the same as that of a system with infinite ranged elastic interactions (up to logarithmic corrections in two dimensions). In other words, the upper critical dimension is equal to the physical dimension of the fault, which is 2 [6,8]. (Some of the static mean-field exponents turned out to be the same as in other quasi-static models [6].) In the presence of small but nonzero weakening effects of amplitude $\epsilon$ a critical rupture size (slipping area) $n_{c r}$ for "runaway" or "characteristic fault size" events was calculated perturbatively [2] and was found to scale as $1 / \epsilon^{2}$. Faults of larger area than this size are expected to display the characteristic earthquake distribution, with small events up to size $n_{c r}$, and no events of intermediate size between $n_{c r}$ and the characteristic fault size events. For faults of smaller total area than $n_{c r}$ only the power law scaling region of the small events is seen, so the distribution is of the Gutenberg Richter type.

In this paper we examine a mean-field model with a range of dynamical weakening effects from weak to strong, and different levels of disorder in the brittle properties. Specifically, we study the model of Ben-Zion and Rice [1], which involves simple approximations of dynamic frictional weakening (similar to static versus dynamic friction), but replace the physical long range elastic interactions with infinite range interactions. In addition to exhibiting both "power law" and "characteristic" scaling of event sizes, this model exhibits the possibility of coexistence of these two types of behavior. That is, for a given set of model parameters, the system has two distinct persistent stationary states. In an infinitely large system it will depend on the initial conditions whether the system displays Gutenberg Richter or charac- 
teristic earthquake type behavior. Faults of finite size can spontaneously switch from one state to the other on time scales that are exponentially large in system size. The switching times (or "persistence times") are determined by nucleation processes from one state to the other, similar to flips back and forth at coexistence in finite thermally equilibrated systems. Many of the qualitative features seem to be sufficiently robust to be applicable to real fault zones. Interesting to note, such "switching" behavior appears to characterize long paleoseismic records observed along the Dead Sea transform fault system in Israel [9], and is compatible with other paleoseismic [10] and geologic [11] data. In addition, qualitatively similar switching has been recently found in regional models of disordered fault systems [12].

The remainder of this paper is organized as follows: In Section II, we define the model and provide a summary of the main results. In Section [II], we present a detailed analysis of the model along with comparisons with numerical simulations. In Section IV, we compare our results with earlier studies of similar models and discuss their potential relevance to natural fault systems modeled as a narrow fault zone in a three dimensional elastic surrounding media.

\section{THE MODEL AND SUMMARY OF RESULTS}

Ben-Zion and Rice [1] suggested that a heterogeneous fault system with offsets and branches may be represented by an array of discrete cells in a two dimensional plane, with spatially varying "macroscopic" constitutive parameters that model the heterogeneity of the original fault system. This model fault on the $(x, z)$ plane can be considered as a collection of brittle patches mapped onto the interface between two tectonic blocks, which move with (small) relative transverse velocity $v \widehat{\mathbf{x}}$ far away from the fault. In the simple realizations used in refs. [1,2], and here, (as in related models [5]), the fault plane is segmented into $N$ geometrically equal cells. In the mean field approximation of infinite range elastic interactions, the local stress $\tau_{i}$ on cell $i$ is given by 


$$
\begin{aligned}
\tau_{i} & =J / N \sum_{j}\left(u_{j}-u_{i}\right)+K_{L}\left(v t-u_{i}\right) \\
& =J \bar{u}+K_{L} v t-\left(K_{L}+J\right) u_{i}
\end{aligned}
$$

where $u_{i}$ is the total fault offset of cell $i$ in the horizontal $(x)$ direction, $\bar{u}=\left(\sum_{j} u_{j}\right) / N, J / N$ is the elastic coupling between cells in the mean-field approximation, and $K_{L}$ is the effective loading stiffness of the bulk material surrounding the fault patch.

Initially, the fault is in a relaxed configuration, i.e. all stresses are less than a local static failure threshold stress $\tau_{s, i}$. In the absence of brittle failures the stresses at the cells increase uniformly due to the external loading and $\dot{\tau}_{i}=K_{L} v$. As long as no cell reaches its failure threshold, $\dot{u}_{i}=0$ everywhere. When the stress at a cell becomes larger than $\tau_{s, i}$, the cell slips by an amount $\delta u_{i}=\left(\tau_{s, i}-\tau_{a, i}\right) /\left(K_{L}+J\right)$, to reduce its stress from $\tau_{s, i}$ to an arrest stress $\tau_{a, i}$. (The nonuniformity of failure and arrest stresses across the fault plane models the spatial heterogeneity of real fault zones [1].) Consequently, during failure cell stresses change by [cf. Eq.(11)]

$$
\begin{aligned}
& \delta \tau_{i}=\tau_{a, i}-\tau_{s, i}, \\
& \delta \tau_{j}=(c / N)\left(\tau_{s, i}-\tau_{a, i}\right), \quad j \neq i,
\end{aligned}
$$

where $c \equiv J /\left(K_{L}+J\right)$ is a "conservation parameter" giving the fraction of the stress drop of the failing cell retained in the system after the slip. As pointed out in Refs. [2,7], for fault zones with characteristic linear dimensions of $O(L)$, the "loading spring constant" is $K_{L} \sim 1 / L$, provided that the stress loading of the fault is either due to uniformly moving (creeping) boundaries or applied forces at distances of $O(L)$ away from the fault plane. For the case $N=L^{2},(1-c) \sim O(1 / \sqrt{N})$. A value $c<1$ for a large system would be physically realized if the external drive is closer to the fault than its linear extent.

During the failure process, the slipped cell is assumed to be weakened by the rupture, such that its failure strength is reduced to a dynamical value $\tau_{d, i} \equiv \tau_{s, i}-\epsilon\left(\tau_{s, i}-\tau_{a, i}\right)$, with $0 \leq \epsilon \leq 1$ parameterizing the relative importance of dynamical weakening effects in the system. If the failure stress transfer brings other cells to their failure threshold, an avalanche 
of cell failures, i.e., "rupture propagation", occurs according to Eqs.(2) until all cells are at stresses $\tau_{i} \leq \tau_{s, i}$ [13]. It is assumed that these avalanches happen on time scales short compared to the external loading time (i.e. $v$ is adiabatically small), so that the external load is kept constant during an earthquake. In time intervals between earthquakes, all cells are assumed to heal completely, thus failure thresholds are reset to their static value $\tau_{s, i}$ and the external loading resumes until the next cell failure.

In order to simplify notation, it is useful to introduce rescaled stress variables

$$
\begin{aligned}
s_{i} & \equiv 1-\frac{\tau_{s, i}-\tau_{i}}{\left\langle\tau_{s, i}-\tau_{a, i}\right\rangle}, \\
s_{a, i} & \equiv 1-\frac{\tau_{s, i}-\tau_{a, i}}{\left\langle\tau_{s, i}-\tau_{a, i}\right\rangle}, \\
s_{d, i} & \equiv 1-\frac{\tau_{s, i}-\tau_{d, i}}{\left\langle\tau_{s, i}-\tau_{a, i}\right\rangle}=1-\epsilon\left(1-s_{a, i}\right),
\end{aligned}
$$

such that cell failure always occurs when $s_{i}=1$, and $\left\langle s_{a}\right\rangle=0$. (Here, \langle\rangle symbolizes averaging over all cells in the fault zone.) The arrest stress $s_{a, i}$ is uncorrelated from cell to cell, and is picked once for each segment from a probability distribution $\rho\left(s_{a}\right)$ with mean 0 and a compact support $(-W / 2, W / 2)$ of width $0 \leq W \leq 2$, which characterizes the heterogeneity of the fault system. [In our simulations we have used the parabolic distribution $\rho\left(s_{a}\right)=3\left(W^{2}-4 s_{a}^{2}\right) /\left(2 W^{3}\right)$, for $-W / 2 \leq s_{a} \leq W / 2$ and 0 otherwise.] Unless stated otherwise, the focus is on the small disorder limit $W \ll 1$ and moderate values for $\epsilon$, which are considered fixed, and the properties of the system are analyzed as a function of varying conservation parameter $c$ and system size $N$. (In the last section of the paper we discuss the effects of larger values of $W$ as well.) The size of an earthquake refers to the number of cells that failed, (i.e. the "area" on the fault that slips in an earthquake).

For $N \rightarrow \infty$, depending on relative values of the system parameters, there are in general two possible steady-state distributions of cell stresses and of earthquake magnitudes. We refer to these as as "phases":

(A) The "Gutenberg-Richter" (G-R) Phase: This phase, possible in both regions 1 and 2 of Fig. 1, is characterized by a distribution of earthquake sizes $p_{e}^{(f)}(n)$ of power law form. In infinite systems $(N \rightarrow \infty)$, it is given by 


$$
p_{e}^{(f)}(n) \approx A_{f} n^{-3 / 2} \exp \left(-n / n_{c f}\right), \quad n \ll N
$$

with a characteristic cutoff size $n_{c f} \approx 2(1-c)^{-2}$ that diverges as $c \nearrow 1$. (Finite size corrections are given in equation (15) below). The stress $s_{i}$ at a given cell is independent of all others and is equally likely to take any allowable value, i.e.,

$$
\operatorname{Prob}\left(s \leq s_{i} \leq s+d s\right)=\frac{d s}{1-s_{a, i}}, \quad s_{a, i} \leq s \leq 1
$$

Thus, the stress distribution in the G-R phase is given by

$$
p^{(f)}(s)=\int_{-\infty}^{s} d s_{a} \frac{\rho\left(s_{a}\right)}{1-s_{a}}
$$

which is uniform and equal to $\bar{p} \equiv\left\langle\left(1-s_{a}\right)^{-1}\right\rangle$ in the interval $(W / 2) \leq s \leq 1$ [see Fig. 2(a), inset]. In this phase each cell fails at most once during an earthquake, and therefore dynamical effects are largely irrelevant. An infinitely large system which started in the G-R phase will remain it this phase. In finite systems $N<\infty$, with parameters in region 2 of figure 1, however, a very large earthquake of size $(1-\epsilon) N / c$ or greater occasionally triggers dynamical effects that lead to a catastrophic "runaway" event in which all cells eventually fail and cause a substantial change in the stress distribution and subsequent evolution of the system, as outlined next.

(B) The "Runaway" Phase: This phase is characterized by a quasi-periodic occurrence of system wide earthquakes in which all cells fail. As a result of dynamical effects, the stress $s_{i}$ in a cell immediately after such a "runaway" event is independent of other cells and is equally likely to take any value between its arrest stress and dynamical failure stress, i.e.,

$$
\operatorname{Prob}\left(s \leq s_{i} \leq s+d s\right)=\frac{d s}{s_{d, i}-s_{a, i}}, \quad s_{a, i} \leq s_{i} \leq s_{d, i} .
$$

The stress distribution is thus given by

$$
p^{(r)}(s)=\frac{1}{1-\epsilon} \int_{\frac{s-(1-\epsilon)}{\epsilon}}^{s} d s_{a} \frac{\rho\left(s_{a}\right)}{1-s_{a}},
$$

which is uniform and equal to $\bar{p} /(1-\epsilon)$ in the interval $(W / 2) \leq s \leq 1-\epsilon-(\epsilon W / 2)$ [see Fig. 2(b), inset]. The runaway event is followed by a quiescent period during which stresses 
on the cells build back up from their dynamic failure value to near their static failure value. Subsequent small events are followed by the next runaway event, at which point the stress distribution is reset to Eq.(10). These background small events have a size distribution similar to events in the G-R phase, but with a different cutoff size. In an infinite system (for finite size corrections see equation (18) below):

$$
\begin{aligned}
p_{e}^{(r)}(n) & \approx A_{r} n^{-3 / 2} \exp \left(-n / n_{c r}\right), \quad n \ll N, \\
n_{c r} & \approx \frac{2(1-\epsilon)^{2}}{(1-\epsilon-c)^{2}},
\end{aligned}
$$

which diverges as $c \searrow(1-\epsilon)$. However, this divergence is never observed, as the runaway phase becomes unstable against breakup into the G-R phase when $c<c^{*} \equiv(1+\epsilon)^{-1}$ for the following reason: If the background small events during a cycle involve at least a fraction $r_{c}$ of the cells, the subsequent large event is unable to cause all of the cells to fail, since the cells that failed during background activity are farther away from their failure stress. This typically causes a spontaneous breakup of the bunched stress distribution and a resumption of the G-R phase. The fraction of cells needed to cause this breakup is given by $r_{c}=1+\epsilon-c^{-1}=\left(c^{*}\right)^{-1}-c^{-1}$ as is derived in section $[1]$. When $c \searrow c^{*}$, the size of background events necessary to cause breakup vanishes and the runaway phase becomes unstable, i.e. for $c<c^{*}$ the G-R phase is the only persistent phase, regardless of the initial conditions. For $c>c^{*}$, the G-R phase and the Runaway phase are both persistent in an infinite system. In an infinite system the initial conditions determine which one the system displays. In a finite system, however, exponentially rare earthquakes can lead to nucleation from the G-R phase into the Runaway phase and vice versa. Equations (16) and (20) below give estimates of the times spent in the respective phases between such nucleation (or "switching") events.

\section{ANALYSIS OF THE MODEL}

The results quoted above have been obtained by mapping earthquakes in the model to corresponding events in a stochastic process, which is approximated by a series of Bernoulli 
trials [14] in order to be able to obtain analytical estimates for the various quantities of interest, such as distributions of earthquake sizes and persistence times for the two phases.

\section{A. Gutenberg-Richter Phase}

Let us first focus on the G-R phase. At some instant $t$ immediately preceding a cell failure, consider the sequence $\left\{X_{n} \equiv 1-s_{i(n+1)}\right\}$, where $i(n)$ is the index of the cell that has the $n$th largest stress in the system [See Fig. 3]. For a large system, the stress gaps $\left\{\delta s_{n}=X_{n-1}-X_{n}\right\}$ are (almost) independent of each other, drawn from an exponential probability distribution, i.e., $\operatorname{Prob}\left(\delta s_{n}=s\right)=\exp (-\bar{p} N s)$, with $\bar{p} \equiv\left\langle\left(1-s_{a}\right)^{-1}\right\rangle$. For $n \gg 1, X_{n}$ resembles a biased random walk with a mean $\mu_{X}(n)=n /(\bar{p} N)$ and variance $\sigma_{X}^{2}(n)=n /(\bar{p} N)^{2}$. As long as dynamical effects are absent, the stress redistributed to each cell following the failure of the first $n$ cells is given by a random variable $Y_{n}$ with mean $\mu_{Y}(n)=n c /(\bar{p} N)$ and variance $\sigma_{Y}^{2}(n) \approx n(c W / \bar{p} N)^{2} \ll \sigma_{X}^{2}(n)$. A triggered earthquake can sustain itself only if the redistributed stresses exceed the stress gaps. Therefore, $Z_{n} \equiv$ $X_{n}-Y_{n}<0$ during an earthquake and it immediately follows that the distribution of earthquake sizes for $N \gg n \gg 1$ are given in terms of the distribution of first passage times of biased random walks. Approximating the continuous probability distribution of the step sizes of $\left\{Z_{n}\right\}$ with a Bernoulli process (where steps of equal size are taken up or down with probability $p$ and $1-p$, respectively), we can utilize results available for Bernoulli trials [14,

$$
\operatorname{Prob}\left(Z_{i}<0,0<i<n ; Z_{n}=0\right)=\frac{\operatorname{Prob}\left(Z_{n}=0\right)}{n},
$$

i.e. the probability for the first return to the origin after $n$ steps equals the total probability of reaching the origin after $n$ steps divided by $n . \operatorname{Prob}\left[Z_{n}=0\right]$ can easily be calculated [14] for $N \gg n \gg 1$. One obtains Eq.(6) with $n_{c f}=2\left(\mu^{2}+\sigma^{2}\right) / \mu^{2}$, where $\mu$ and $\sigma^{2}$ are the mean and variance of the step size for the process $Z_{n}$, respectively. Substituting the values $\mu=(1-c) /(\bar{p} N)$ and $\sigma^{2} \approx 1 /(\bar{p} N)^{2}$, the cutoff length is given by

$$
n_{c f} \approx 2\left(1+(1-c)^{-2}\right)=\frac{2}{(1-c)^{2}}\left\{1+O\left[(1-c)^{2}\right]\right\},
$$


where the last approximation is justified since treating $Z_{n}$ as a Bernoulli process is expected to yield relative errors of $O\left(\mu^{2} / \sigma^{2}\right)$.

For finite-sized systems, when the fraction of failed cells $r=n / N$ is no longer small, Eq.(6) needs to be modified since the stress gaps are not entirely independent: In order to correctly reflect the fact that $Z_{N}=1-c$ to within $O(1 / N)$, the Bernoulli process should be constrained to return to its mean value after $N$ steps. This can be achieved by calculating the corresponding conditional probabilities:

$$
\begin{aligned}
p_{e}^{(f)}(n) & =\frac{\operatorname{Prob}\left(Z_{n}=0\right)}{n} \frac{\operatorname{Prob}\left(Z_{N-n}=1-c\right)}{\operatorname{Prob}\left(Z_{N}=1-c\right)} \\
& =\frac{\tilde{A}_{f}}{n^{3 / 2}} \exp \left\{-\frac{n(1+n / N)}{n_{c f}}\right\},
\end{aligned}
$$

which reduces to Eq.(6) in the limit $n \ll N$. ( $\tilde{A}_{f}$ is a constant fixed by normalization.) Figure 2(a) shows the distribution of event sizes for numerical simulations of the model with $N=400$, for values $c=0.6,0.7$ and 0.8 . (In all presented simulation results, $W=2 / 19$ and $\epsilon=0.5$.) The continuous lines are one-parameter fits to the form (15). The discrepancy between the fitted and theoretical [from Eq.(14)] values of $n_{c f}$ is consistent with the expected relative error.

As mentioned earlier, the failure of all the remaining cells becomes very likely once $(1-\epsilon) N / c$ cells have failed, since the initially failed cells reach their dynamical failure stress. The mean event size is roughly equal to $n_{c f}^{1 / 2}$, therefore the mean time between events is $T_{0} n_{c f}^{1 / 2} / N$, where $T_{0} \equiv\left\langle\tau_{s, i}-\tau_{a, i}\right\rangle /\left(K_{L} v\right)$ is the characteristic time over which a cell is loaded from its arrest stress to its failure stress. The typical waiting time to see a switch to the runaway phase yields [cf. Eq.(15)]

$$
T_{f \rightarrow r} \approx T_{0} \frac{C_{f r} N^{1 / 2}}{n_{c f}^{1 / 2}} \exp \left\{\frac{(1-\epsilon)(1-\epsilon+c)}{c^{2} n_{c f}} N\right\},
$$

with $C_{f r}$ a factor of order unity which varies weakly with $\epsilon$ and $c$ in the region of interest, provided that each attempt is statistically independent of each other. We verified that in our simulations indeed no time correlations of event sizes were present, out to many times the characteristic time $T_{0}$ (see also the discussion section). The distribution of persistence 
times should then obey Poisson statistics with mean $T_{f \rightarrow r}$. Figure 1 depicts the distribution of persistence times (with a fit to Poisson statistics) for $N=400$ and $c=0.73$. Mean persistence times depend very sensitively on the conservation parameter $c$, as shown in the inset of Fig. 团 [15].

\section{B. Runaway Phase}

Let us next consider the runaway phase. Immediately preceding the first cell failure after a runaway event, the stress gaps $\left\{\delta s_{n}=X_{n-1}-X_{n}\right\}$ have the probability distribution $\operatorname{Prob}\left(\delta s_{n}=s\right)=\exp [-\bar{p} N s /(1-\epsilon)]$. Hence, $\left\{X_{n}\right\}$ has a mean $\mu_{X}(n)=n(1-\epsilon) /(\bar{p} N)$ and variance $\sigma_{X}^{2}(n)=n(1-\epsilon)^{2} /(\bar{p} N)^{2}$. As long as dynamical effects are absent, the stress redistributed to each cell following the failure of the first $n$ cells is still given by $\left\{Y_{n}\right\}$ with mean $\mu_{Y}(n)=n c /(\bar{p} N)$ and variance $\sigma_{Y}^{2}(n) \approx n(c W / \bar{p} N)^{2} \ll \sigma_{X}^{2}(n)$. Thus, the mean and variance of the step size for $\left\{Z_{n}\right\}$ is $\mu=(1-\epsilon-c) /(\bar{p} N)$ and $\sigma^{2} \approx(1-\epsilon)^{2} /(\bar{p} N)^{2}$. The probability for an earthquake to terminate after $n$ cell failures is [including finite size corrections in analogy with Eq.(15)]

$$
\begin{aligned}
p_{e}^{(r)}(n) & \approx \frac{\tilde{A}_{r}}{n^{3 / 2}} \exp \left\{-\frac{n(1+n / N)}{n_{c r}}\right\}, \\
n_{c r} & =\frac{2(1-\epsilon)^{2}}{[c-(1-\epsilon)]^{2}}\left\{1+O\left([c-(1-\epsilon)]^{2}\right)\right\}, \quad c>1-\epsilon .
\end{aligned}
$$

Since $\mu<0$ for $1-\epsilon<c, Z_{n}<0$ with finite probability for all $n$ and a runaway event occurs. In fact, a runaway event is inevitable since $Z_{N}<0$ and the runaway event will commence once $\left\{Z_{n}\right\}$ reaches its maximum. The total number of cells that fail before a runaway event is given by the position of the maximum of $\left\{Z_{n}\right\}$, whose probability distribution is proportional to $n_{c r} p_{e}^{(r)}(n)$ for $n \gg n_{c r}$. The mean number of these "precursor" cells is of order $n_{c r}^{1 / 2}$, which remains a finite constant as $N \rightarrow \infty$, i.e., for big systems almost all the slip happens during the runaway events [16].

The remaining cells will all fail during the runaway event. Imagine a situation where a fraction $r>(1-\epsilon) / c$ of the cells have failed. At that point, the total redistributed stress 
per cell, is

$$
\begin{aligned}
S & =c\left[r+\left(r-\frac{1-\epsilon}{c}\right)\left\{c+c^{2}+\cdots\right\}\right] \\
& =\frac{c[r-(1-\epsilon)]}{1-c},
\end{aligned}
$$

where the second term arises from repeat failures of some cells. $S \geq 1$ is needed to ensure that small event cells fail again and recreate the stress distribution (10). This is achieved if

$$
r \geq r^{*} \equiv \frac{1}{c}-\epsilon
$$

Thus, the large event cannot recreate the stress distribution (10) if more than $\left(1-r^{*}\right) N=$ $r_{c} N$ cells fail during background activity. This usually leads to a breakup of the bunched stress distribution and subsequent evolution towards the G-R stress distribution (8). The typical persistence time of the Runaway phase before a switch to the G-R phase is 15

$$
T_{r \rightarrow f} \approx T_{0} \frac{C_{r f} N^{3 / 2}}{n_{c r}^{2}} \exp \left\{\frac{\left(c-c^{*}\right)\left[1+\left(c-c^{*}\right) /\left(c^{*} c\right)\right] N}{c^{*} c n_{c r}}\right\}, \quad c>c^{*},
$$

provided that all attempts are statistically independent of each other. (We have explicitly checked in the simulations that in the runaway phase, the particular realizations of stress distributions immediately following a large event are statistically independent of each other [18]). $T_{r \rightarrow f}$ becomes comparable to the typical time between runaway events when $c \searrow c^{*}$, as expected [17]. Figure 4 depicts the distribution of persistence times and a fit to Poisson statistics for $N=100$ and $c=0.73$. The inset shows the dependence of mean persistence times on $c$ for $N=100$. Although agreement with Eqs. (16) and (20) is rather poor, the strong exponential dependence as a function of conservation parameter is evident.

\section{DISCUSSION}

The persistence times in both the G-R phase and the runaway phase diverge exponentially with system size for $(1+\epsilon)^{-1}<c<1$, and the system remains in either phase for

extremely long times, thus the phase space has two almost stable attractors. Clearly, the 
runaway phase represents a more "ordered" stress distribution. Indeed, the basin of attraction for the runaway phase is extremely small. In order to quantify this aspect, consider the "configurational entropy" for a given stress distribution $p(\tilde{s})$, with $\tilde{s}_{i} \equiv\left(\tau_{i}-\tau_{a, i}\right) /\left(\tau_{f, i}-\tau_{a, i}\right)$ :

$$
S_{\text {conf }}(\{p\}) \equiv-\int d \tilde{s} p(\tilde{s}) \ln [p(\tilde{s})] .
$$

For the G-R phase, $S_{\text {conf }}^{(f)}=0$, indicating that a "generic" stress distribution characterizes the G-R phase. On the other hand, in the runaway phase

$$
S_{\text {conf }}^{(r)}=-\int d \tilde{s} p^{(r)}(\tilde{s}) \ln \left[p^{(r)}(\tilde{s})\right]=\ln (1-\epsilon)
$$

indicating that the stress distribution is highly organized in that phase. For discrete $N$ the stress distribution is approximated by a histogram of the stress values, and the integral is replaced by the sum over all bins of the histogram.

The time evolution of the configurational entropy of the stress distribution, calculated with a 10 bin histogram, is depicted in Fig. 0 along with event sizes. It is clear that $S_{\text {conf }}(t)$ can be used as an "order parameter", a number that distinguishes the G-R phase and the runaway phase, with the advantage that it can be determined at any instant. Histograms of event sizes require a finite time interval to collect, and there is always the danger of mixing events from one phase with the other, thereby confusing the picture: The cumulative event size distribution over many persistence times is a weighted average of two entirely different event distributions, which obscures the underlying physical phenomena. $S_{\text {conf }}$ provides a reliable way to separate the two phases and makes it possible to accumulate accurate event size distributions for both of them. Unfortunately, such a quantity cannot be determined from existing field data since the spatial distribution of stress is unknown.

So far, the discussion has centered around the $W \ll 1$ limit, and the main role played by the heterogeneities has been the "randomization" of the stress distribution at time scales over which all cells fail a few times. The distribution of loading times, over which the cells are loaded from the individual arrest stress to the failure stress, has a mean $T_{0} \equiv$ $\left\langle\tau_{s, i}-\tau_{a, i}\right\rangle /\left(K_{l} v\right)$ and standard deviation of order $W T_{0}$. Therefore, the "randomization" 
time, over which the stress variables $s_{i}$ become roughly uncorrelated, is of order $T_{0} / W$. Thus, even for small $W$, for large enough $N$ this will be small compared to the persistence times, which scale exponentially in $\mathrm{N}$ [See Eqs.(16) and (20)]. This ensures the consistency of the assumption that the earthquakes are basically statistically independent of each other. The validity of this assumption of statistical independence can be explicitly verified by examining the time correlations of event sizes numerically; indeed no trace of any correlation was found in our simulations, out to many times the randomization time $T_{0} / W$. Likewise, we have explicitly checked that in the runaway phase, the particular realizations of stress distributions immediately following a large event are statistically independent of each other [18].

For finite values of $W<2$, we expect most of the features to remain qualitatively unchanged: In the G-R phase, the exponential cutoff size still diverges as $n_{c f} \sim(1-c)^{-2}$, and although $c^{*}$ in general depends on $W$ and the shape of $\rho\left(s_{a}\right)$, there is still a persistent runaway phase for $c^{*}<c \leq 1$. However, the situation is likely to change qualitatively once arrest stresses can be arbitrarily close to failure stresses, i.e. $W=2$, and new values for the arrest stresses are picked every time a cell fails [19]. This corresponds to the situation discussed in Ref. [2] for finite-dimensional systems. Immediately upon introduction of dynamical weakening $(\epsilon>0), n_{c f} \sim \epsilon^{-2}$ when $c$ approaches 1, i.e. the cutoff size no longer diverges. Furthermore, for $c=1$ the G-R phase is no longer persistent since the persistence time $T_{f \rightarrow r}$ remains finite for large $N$.

Some of the results presented for the mean-field model, especially the qualitative phase diagram, calculated exponents for the power law earthquake distributions, and the divergence of the cutoff length scale, can be expected to apply to models with realistic interactions, up to logarithmic corrections. Such is because the underlying critical points that control these exponents remain mean-field-like down to 2 dimensional faults. This result is firmly established for the $\epsilon=0$ case [2:80]. At finite $\epsilon$ one expects the nucleation size for the runaway phase, which equals $(1-\epsilon) N / c$ in mean field theory, to become independent of the system size, since elastic forces in the fault plane concentrate stresses along the earthquake rupture 
front as the earthquake progresses. Earthquakes bigger than a finite nucleation size $N_{\text {crack }}$ become unstoppable in the presence of dynamic weakening effects and small disorder [1], and rupture the entire fault. Nevertheless, for $n_{c f}<N_{c r a c k}$, the mean-field scaling results may still apply at finite $\epsilon$, provided that $W<2$, i.e., there is a finite minimum stress drop associated with each cell failure. For systems with $N_{\text {crack }}>N$, this range will extend all the way to the fault size. In this case, one remarkable consequence is that since generically $(1-c) \sim 1 / \sqrt{N}$ [2, 6], the cutoff size in the G-R phase $n_{c f} \sim(1-c)^{-2} \sim N$, i.e., earthquakes on individual fault zones obey power law statistics for events up to a finite fraction of the entire system size.

An important result is the possibility that a fault system might switch from a "Gutenberg-Richter" earthquake distribution spontaneously to a "characteristic" earthquake distribution, as in the mean-field model. We note that calculations based on an entirely different model, simulating the coupled evolution of regional earthquakes and faults in a rheologically layered $3 D$ solid [12], show similar behavior. Clear observation of such mode switching in nature requires data sets spanning many thousands of years. Paleoseismic studies attempt to construct long histories of seismic events at given locations from sequences of displaces and highly disturbed rock layers. Remarkably, the longest available paleoseismic records, documenting large earthquake activity along the Dead sea transform in Israel [9] appear to be characterized by alternating phases of intense seismic activity lasting a few thousands of years, and periods of comparable length without large seismic events. Other, qualitatively similar alternating deformation phases have been documented in the eastern CA shear zone [10] and the Great Basin Province in the western US [11].

Another intriguing possibility might arise in a fault system of weakly coupled segments driven under similar conditions. The seismic response of such systems might exhibit a sort of "coexistence", i.e., a fraction of the patches might follow characteristic scaling whereas the others obey Gutenberg-Richter scaling, giving rise to a hybrid event size distribution. This may explain examples in the data of reference [4], where the characteristic "bump" in the distribution was not very pronounced. Finally, we note that part or all of the low magnitude 
seismicity in the G-R phase may be too small to be detected by a seismic network. In this case the spontaneous switching between the Runaway and G-R phases may be interpreted as transitions from seismic response of a fault system to creep-like behavior.

\section{ACKNOWLEDGMENTS}

We have greatly benefited from extensive discussions with Daniel S. Fisher, Jim Rice, and Sharad Ramanathan.

KD gratefully acknowledges support from the Society of Fellows of Harvard University, and NSF via DMR 9106237, 9630064, Harvard's MRSEC and Harvard's Milton Fund. DE acknowledges support by the NSF through Grants No. DMR-9106237, No. DMR-9417047, and No. DMR-9416910. YBZ was supported by the Southern California Earthquake Center (based on NSF Cooperative Agreement EAR-8920136 and USFS Cooperative Agreement 14-08-0001-A0899). 


\section{REFERENCES}

* Present Address: Exxon Research And Engineering, Clinton Twp., Rte. 22 East, Annandale, New Jersey 08801. Electronic Address: mdertas@erenj.com.

[1] Y. Ben-Zion and J.R. Rice, J. Geophys. Res. 98 , 14109, 1993; J. Geophys. Res. 100, 12959, 1995; Y. Ben-Zion J. Geophys. Res. 101, 5677 (1996).

[2] D.S. Fisher, K. Dahmen, S. Ramanathan, and Y. Ben-Zion, Phys. Rev. Lett. 78, 4885 (1997).

[3] B. Gutenberg, and C.F. Richter, Ann. Geophys. 9, 1 (1956). The development and dynamics of fault systems has been studied as a non-equilibrium critical phenomenon by e.g.: K. Chen, P. Bak, and S.P. Obukhov, Phys. Rev. A 43, 625 (1991); P. Miltenberger, D. Sornette and C. Vanneste, Phys. Rev. Lett. 71, 3604 (1993); P. A. Couie, C. Vanneste, and D. Sornette, J. Geophys. Res. 98, 21809 (1993).

[4] S.G. Wesnousky Bull. Seismol. Soc. Am. 84, 1940, 1994; M.W. Stirling, S.G. Wesnousky, and K. Shimazaki, Geophys. J. Int., 123, 833 (1996).

[5] Burridge and Knoppoff, Bull. Seismol. Soc. Am 57, 341 (1967); J.M. Carlson, J.S. Langer, B.E. Shaw, Rev. Mod. Phys. 66, 658, 1994, and references therein; C.R. Myers, B.E. Shaw, J.S. Langer, Phys. Rev. Lett. 77, 972 (1996); J.B. Rundle, W. Klein, S. Gross Phys. Rev. Lett. 764285 (1996); D. Cule, T. Hwa Phys. Rev. Lett. 77, 278 (1996).

[6] K. Chen, P. Bak, and S.P. Obukhov, Phys. Rev. A 43, 625 (1991)

[7] K. Christensen and Z. Olami, Phys. Rev. A 46, 1829 (1992) and references therein.

[8] D. Ertaş and M. Kardar, Phys. Rev. E 49, R2532 (1994).

[9] S. Marco, M. Stein, A. Agnon, and H. Ron, J. Geophys. Res. 101, 6179 (1996).

[10] T. Rockwell, personal communication.

[11] R.E. Wallace, Bul. Seismol. Soc. Am. 77, 868 (1987). 
[12] Y. Ben-Zion, V. Lyakhovsky, and A. Agnon, Proc. of 1997 Annual SCEC meeting, 55 (1997); V. Lyakhovsky, Y. Ben-Zion, and A. Agnon, paper in preparation.

[13] Note that cell stresses do not necessarily get arrested at $\tau_{a, i}$ during an earthquake; which would have required the slip amount to be $\left(\tau_{i}-\tau_{a, i}\right) /\left(K_{L}+J\right)$ instead. We use Eqs.(22) in order to mimic continuous time. This choice also makes the model Abelian, i.e. whether a given cell will fail during an earthquake does not depend on which order they fail, simplifying the analysis.

[14] W. Feller, An Introduction to Probability Theory and Its Applications, Vol. 1, Second Ed., Chapter XI (John Wiley \& Sons, Inc., New York, 1957.)

[15] The estimate for the persistence time does not take into account possible two or many step channels to nucleate from one phase into the other. Such corrections, however, are not expected to change the exponential dependence of the persistence time on the system size, i.e. the metastability of the two phases in the considered parameter regime.

[16] For larger disorder $W$ there are yet another kind of background events. Their number is proportional to $N$. In the regime considered here (small $W$, and moderate $N$ ), however, those effects are negligible, as is also confirmed by our simulation results.

[17] Note that $n_{c r}$ remains finite when $c \searrow c^{*}$, therefore the cutoff size for background small events never diverges; the underlying "critical point" is obscured by the breakup instability.

[18] One exception to the statistical independence of events happens during the breakup of the runaway stress distribution, where atypically large (for the G-R phase) earthquakes occur within a few $T_{0}$ of the initiation of breakup. These events should not be included in an analysis of the event size distributions, since they are simply transients and not representative of events in steady-state.

[19] The nature of the heterogeneities in our model differs significantly from those considered 
in Ref. [2], in that they are long-range in the displacements $u$, although short-range in position. This is the physically relevant situation when typical slips of a cell are smaller than the linear cell size, so that substantially, same portions of the the two plates face each other before and after a slip. In reference [12], the heterogeneities evolve as a function of the deformation in a damage rheology model. 


\section{FIGURES}

FIG. 1. Schematic phase diagram of the system. There is a "coexistence" of two persistent stationary states called Gutenberg-Richter and Runaway phases, in a finite region of parameter space, marked region "(2) meta-stable". For region 1 given by $c<c^{*}=1 /(1+\epsilon)$ (line AB) one finds only small avalanches, i.e. the system is always in the Gutenberg-Richter phase.

FIG. 2. Histograms of event size distributions in the two stationary states (phases), for $W=2 / 19, \epsilon=0.5, N=400$. (a) The "Gutenberg-Richter" phase, characterized by a power-law earthquake distribution with an exponential cutoff. Solid lines are fits to the analytic form (15)

with $n_{c f}$ as a fitting parameter. Also indicated are analytic estimates $n_{c f}^{(t h)}$. The inset shows a typical stress distribution of this phase for $c=0.7$. The solid line is a fit to the analytic form (8). The nonuniform region near $s=0$ extends from $-W / 2$ to $W / 2$. (b) The "runaway" phase, with a similar background distribution and large characteristic events. The inset shows a typical stress distribution for $c=0.8$. The solid line is a fit to the analytic form (10). The nonuniform region near $s=0$ extends from $-W / 2$ to $W / 2$. Near $s=\epsilon$ it extends from $\epsilon(1-W / 2)$ to $\epsilon(1+W / 2)$.

FIG. 3. The process $\left\{Z_{n}\right\}$, which shows the incremental amount of stress needed to keep an earthquake going (see text for the precise definition). Each failure event corresponds to a segment of the process that starts out from a maximum up to that point and ends when it exceeds that level, and is marked as alternating circles and squares. The sample shown here, which corresponds to the stress distribution shown in the inset of Figure 2(a), depicts events of size $6,14,2,1,1, \cdots$. The fault is loaded adiabatically between these events, during the intervals when $\left\{Z_{n}\right\}$ moves monotonically up from one maximum to the next. These are shown as dotted lines connecting consecutive events. 
FIG. 4. Distribution of persistence times $T_{f \rightarrow r}$ and $T_{r \rightarrow f}$ for $W=2 / 19, \epsilon=0.5, N=100$, $c=0.73$. The lines are fits of the cumulative probabilities to the Poisson distribution. (Simulations for systems with other parameters that allowed for many more switches during the simulated times clearly also gave Poisson distributions for the distribution of persistence times.) Inset: The dependence of persistence times on conservation parameter $c$, (triangles $T_{f \rightarrow r}$, circles $T_{r \rightarrow f}$ ) for the same values of $W, \epsilon$, and $N$. Statistical errors are comparable to symbol sizes.

FIG. 5. Sample time series of earthquake sizes (top), plotted together with the conformational entropy $S_{\text {conf }}(t)$ (bottom) for $W=2 / 19, \epsilon=0.5, N=100, c=0.73$. (For the calculation of $S_{\text {conf }}(t)$, the simulated stress distribution was approximated by a 10 bin histogram of the stress values, which was evaluated immediately after each earthquake.) The earthquake size distribution changes drastically every time $S_{\text {conf }}$ toggles from 0 to $\ln (1-\epsilon)$, indicating a transition from one phase to the other. A failed switching attempt from the G-R phase to the runaway phase is seen at about $T / T_{0}=10900$. 


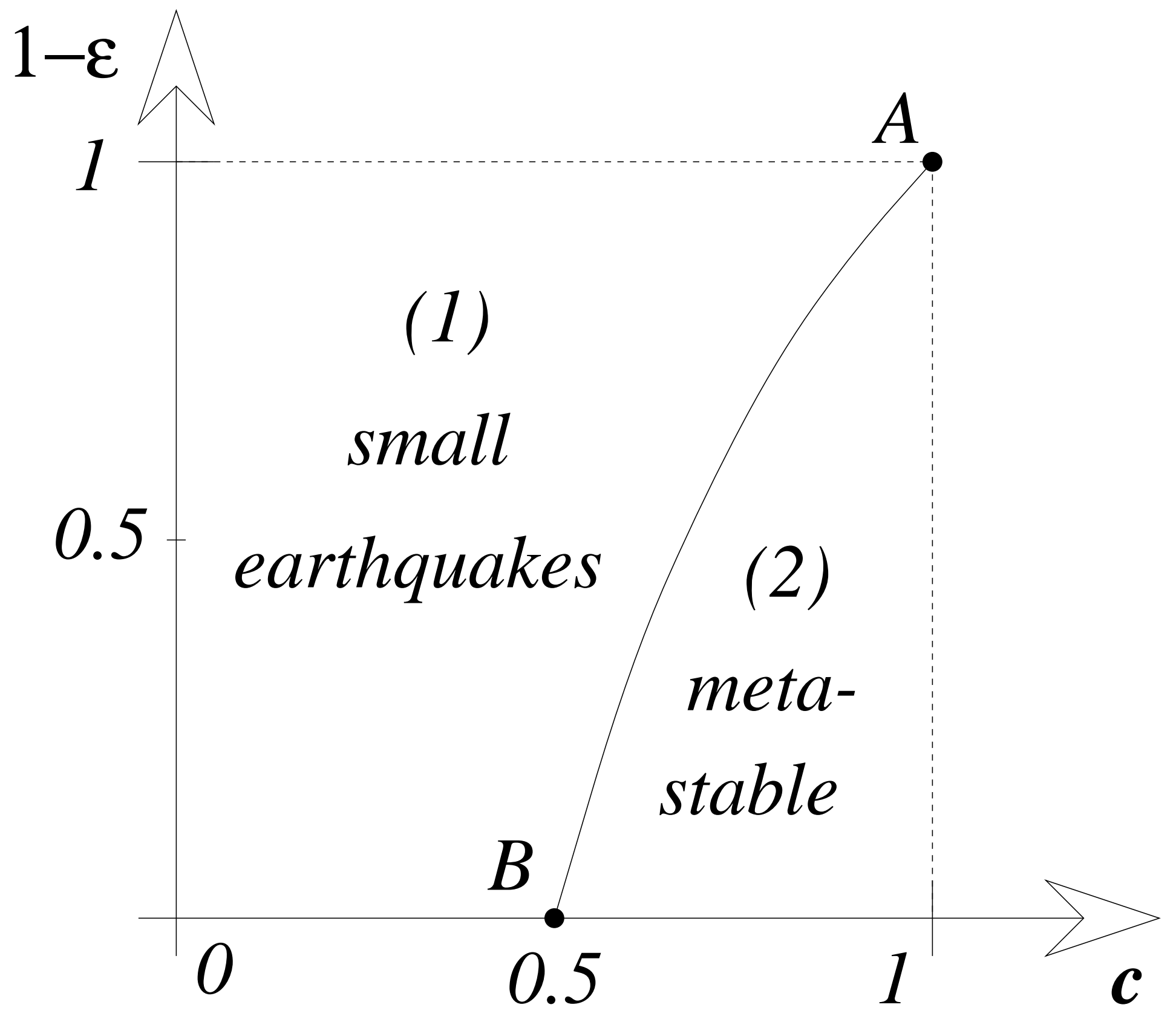




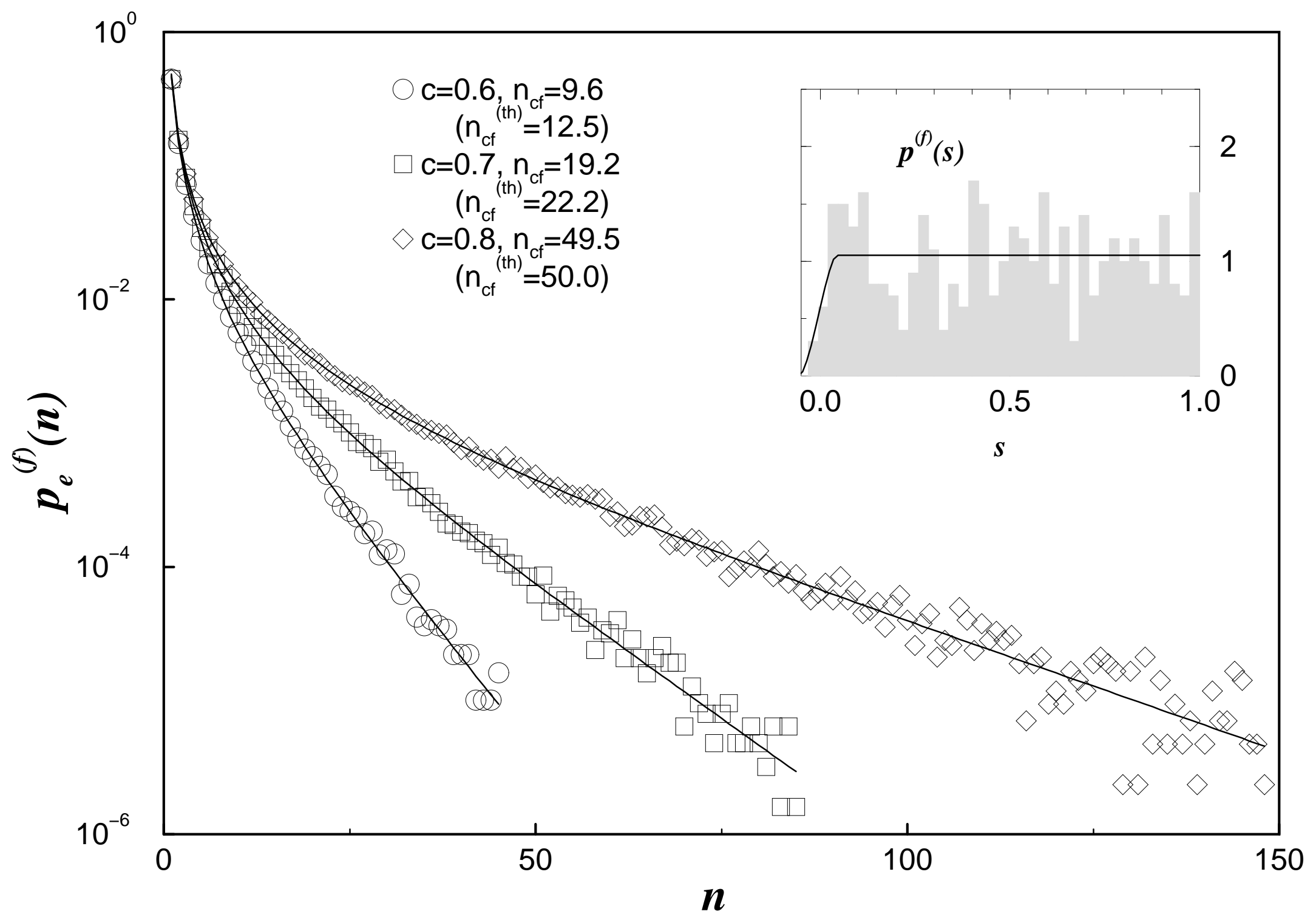




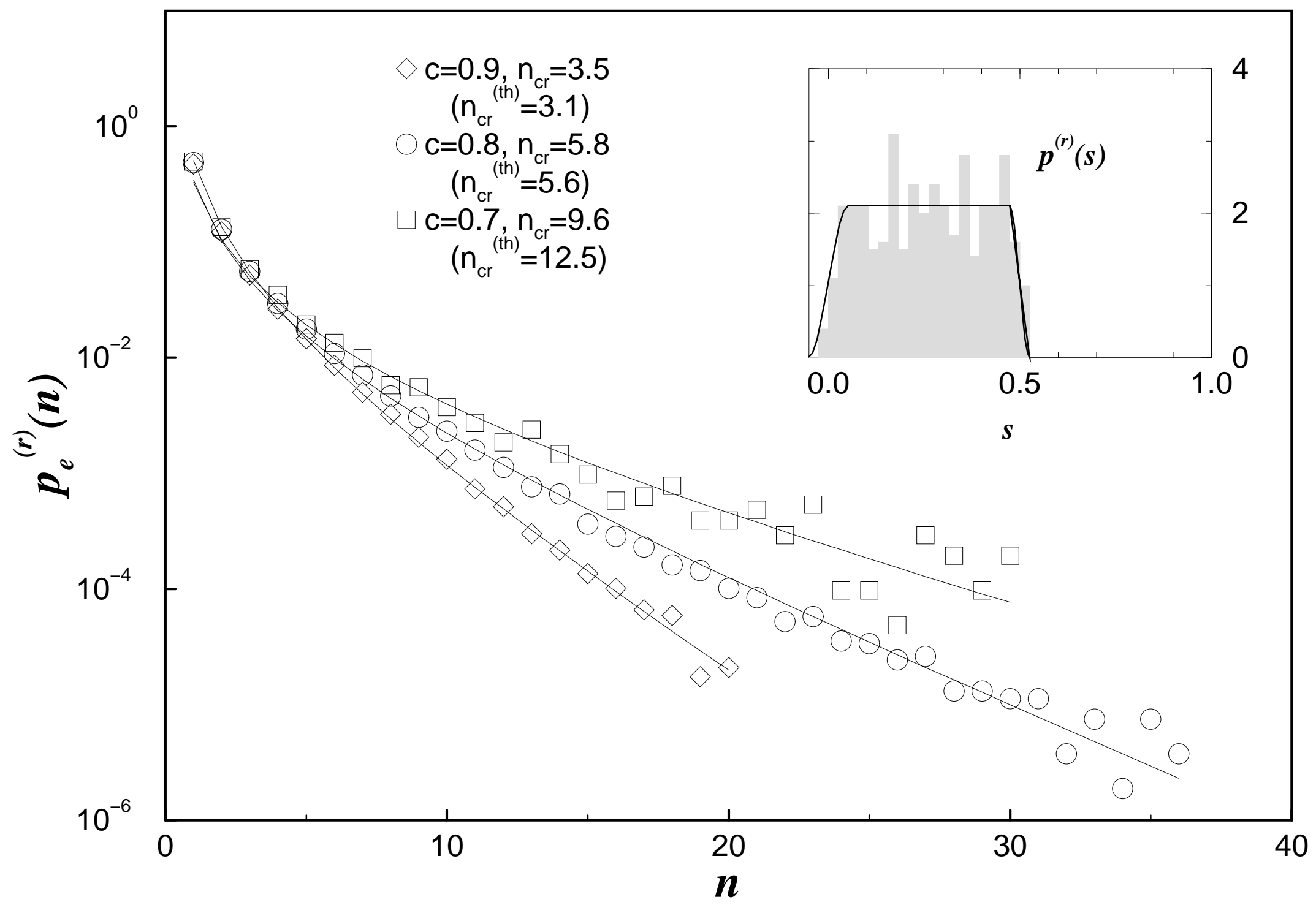




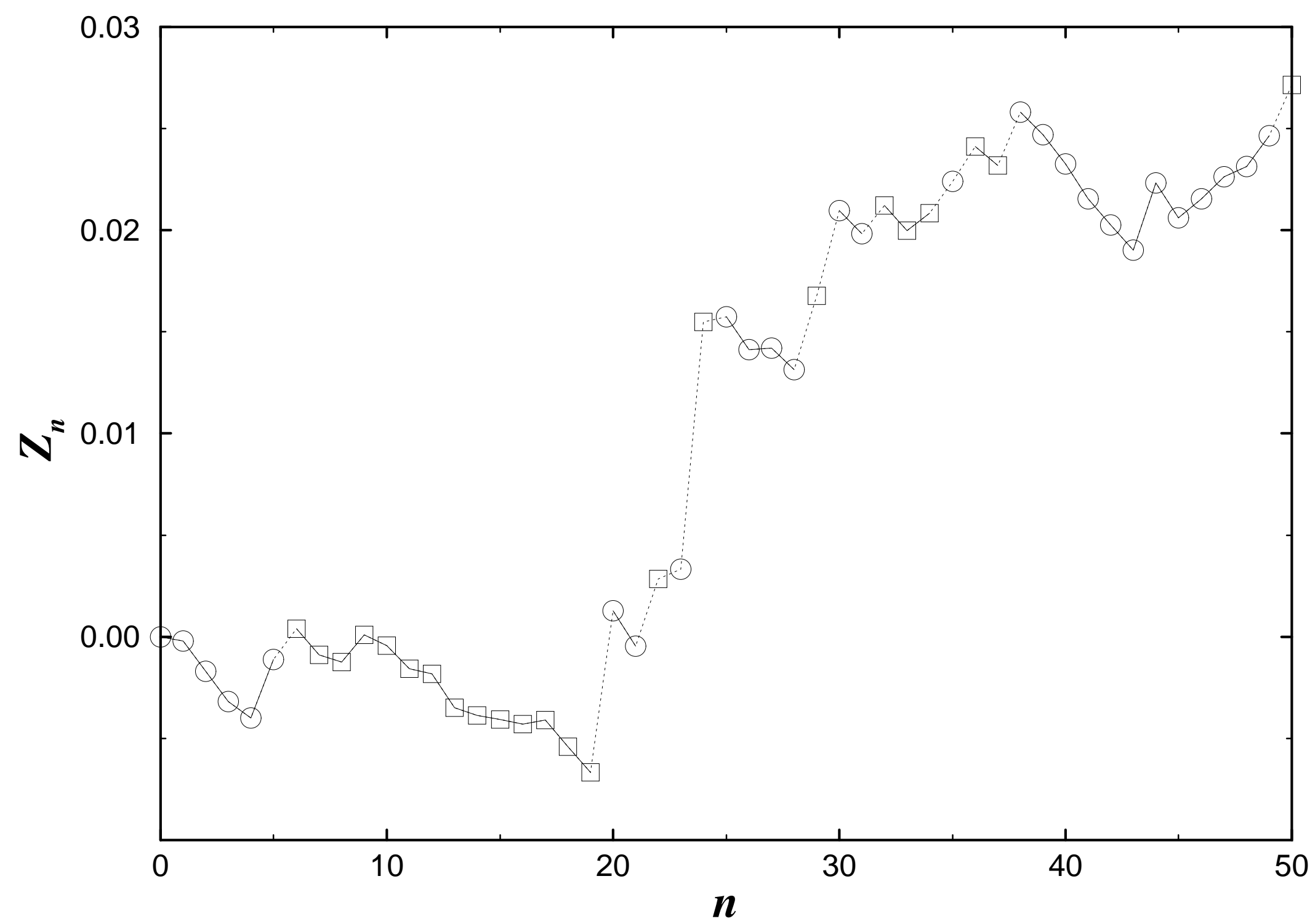




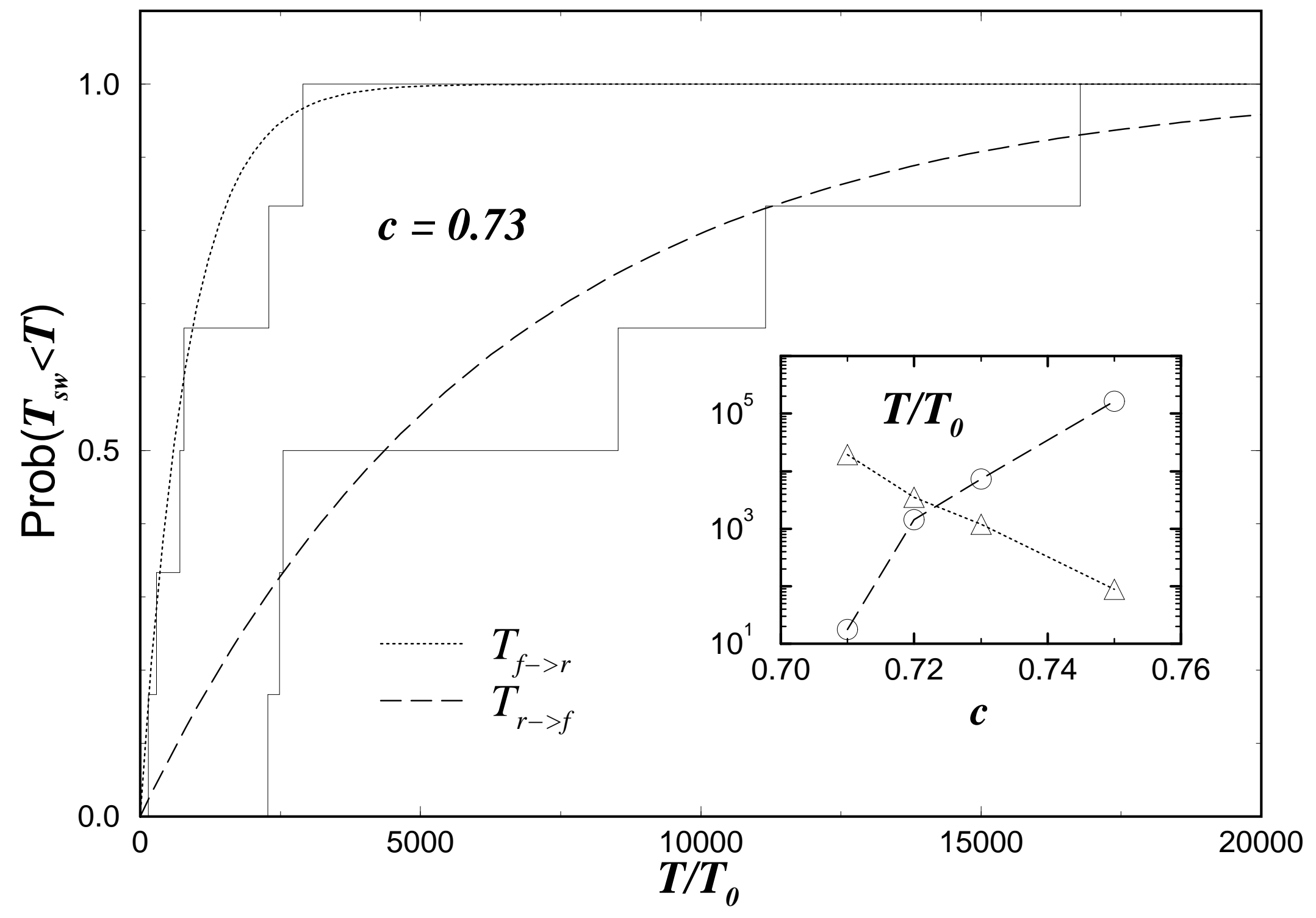



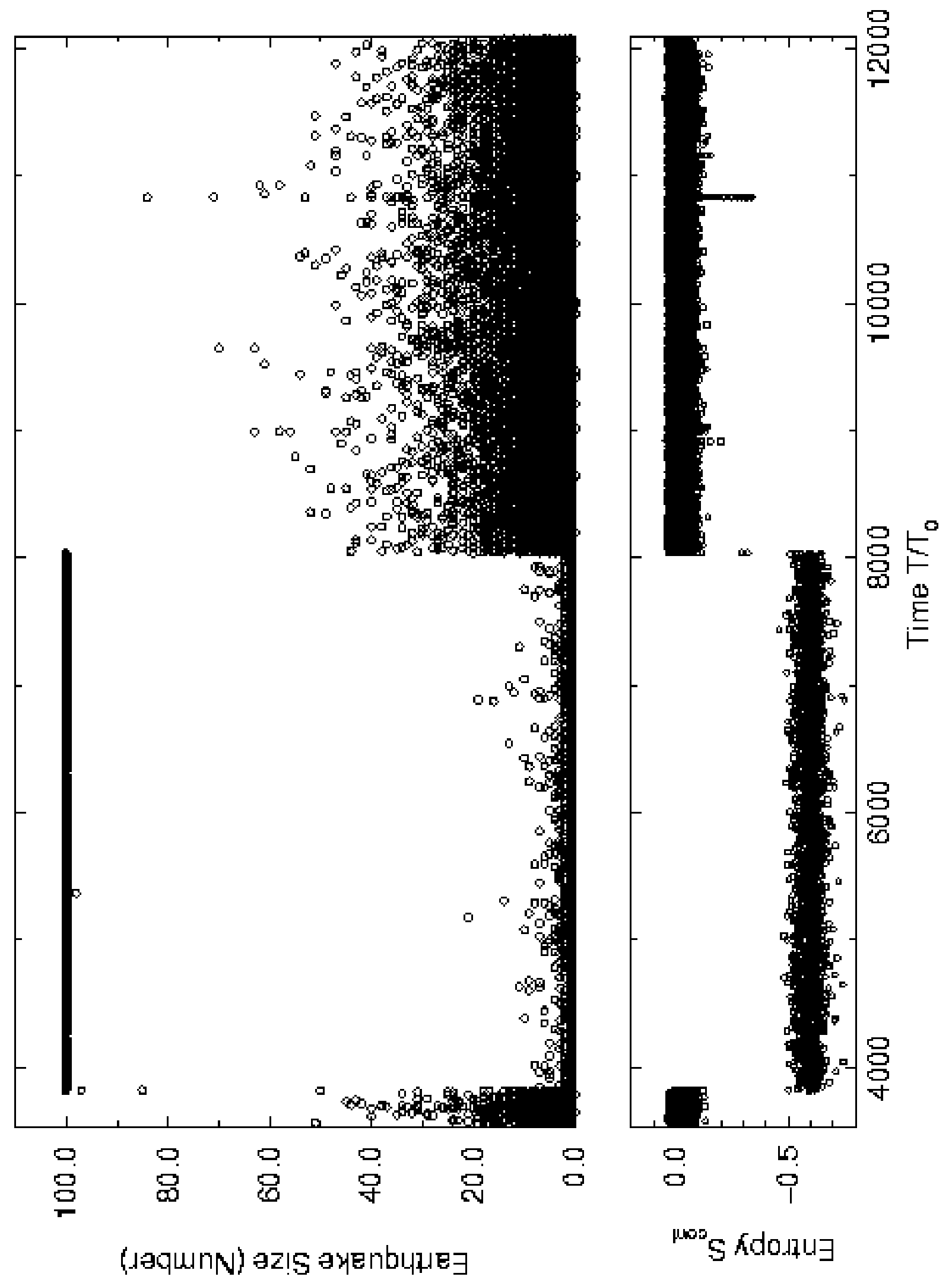